University of Nebraska - Lincoln

DigitalCommons@University of Nebraska - Lincoln

Faculty Publications: Materials Research

Materials Research Science and Engineering

Science and Engineering Center

Center

$9-1-2003$

\title{
Finite-Temperature Anisotropy of PtCo Magnets
}

\author{
Ralph Skomski \\ University of Nebraska-Lincoln, rskomski2@unl.edu \\ Arti Kashyap \\ University of Nebraska-Lincoln, akashyap@Inmiit.ac.in \\ David J. Sellmyer \\ University of Nebraska-Lincoln, dsellmyer@unl.edu
}

Follow this and additional works at: https://digitalcommons.unl.edu/mrsecfacpubs

Part of the Materials Science and Engineering Commons

Skomski, Ralph; Kashyap, Arti; and Sellmyer, David J., "Finite-Temperature Anisotropy of PtCo Magnets" (2003). Faculty Publications: Materials Research Science and Engineering Center. 24.

https://digitalcommons.unl.edu/mrsecfacpubs/24

This Article is brought to you for free and open access by the Materials Research Science and Engineering Center at DigitalCommons@University of Nebraska - Lincoln. It has been accepted for inclusion in Faculty Publications:

Materials Research Science and Engineering Center by an authorized administrator of DigitalCommons@University of Nebraska - Lincoln. 


\title{
Finite-Temperature Anisotropy of PtCo Magnets
}

\author{
R. Skomski, A. Kashyap, and D. J. Sellmyer
}

\begin{abstract}
The temperature dependence of the magnetocrystalline anisotropy of PtCo and its atomic origin are investigated by first-principle and model calculations. The Pt spin moment necessary to realize the leading $5 \mathrm{~d}$ anisotropy contribution is due to neighboring Co atoms. At finite temperatures, Co spin disorder strongly reduces the $\mathrm{Pt}$ moment and the anisotropy. This is in contrast to the situation encountered in $\mathbf{3 d}$ and $\mathbf{3 d}-\mathbf{4 f}$ magnets, where the atomic magnetic moments remain largely conserved, even above the Curie temperature. A consequence of the $L 1_{0}$ mechanism is that the $K_{1}(T)$ curve of exhibits a negative curvature, in contrast to the unfavorable positive curvature for rare-earth transition-metal magnets.
\end{abstract}

Index Terms-Anisotropy, finite-temperature magnetism, inteatomic exchange, $\mathrm{L} 1_{0}$ magnets, permanent magnets, PtCo.

\section{INTRODUCTION}

$\mathbf{T}$ ETRAGONAL intermetallics having in the $\mathrm{L} 1_{0}$ structure, such as PtCo, PdFe, and PtFe, have attracted much attention as permanent magnets and, more recently, in the field of magnetic recording [1]-[6]. One reason is the comparatively large magnetocrystalline anisotropy, about $4.9 \mathrm{MJ} / \mathrm{m}^{3}$ for PtCo. The magnets are characterized by the simultaneous involvement of $3 \mathrm{~d}$ and $5 \mathrm{~d}$ (or $4 \mathrm{~d}$ ) atoms, which distinguishes them from 3d-based permanent magnets, such as alnico and barium ferrite, and from rare-earth transition-metal intermetallics, where specific tasks are realized by $4 \mathrm{f}$ and $3 \mathrm{~d}$ atoms. The zero-temperature properties of $\mathrm{L} 1_{0}$-type layered structures are reasonably well understood [7], [8], but the investigation of their finite-temperature behavior is still in its initial stage. This refers, in particular, to the magnetic anisotropy. From an experimental point of view, it is difficult to control the composition and crystallinity over a wide temperature range, and competing phases such as disordered fcc have a pronounced effect on the anisotropy. Here, we focus on the theoretical understanding of the temperature dependence of the magnetic anisotropy.

Typical uniaxial transition-metal magnets, such as $\mathrm{YCo}_{5}$, exhibit a nearly linear decrease of the first anisotropy constant $\mathrm{K}_{1}$ as a function of temperature, and the mean-field prediction for the anisotropy of classical one-sublattice magnets is $\mathrm{K}_{1}(\mathrm{~T})=$ $\mathrm{K}_{1}(0)\left(1-\mathrm{T} / \mathrm{T}_{\mathrm{c}}\right)$. Akulov's low-temperature $\mathrm{n}(\mathrm{n}+1) / 2$ power

Manuscript received January 6, 2003. This work is supported in part by the Department of Energy (DOE), in part by the Army Office of Scientific Research (AFOSR), in part by National Science Foundation (NSF) Materials Research Science and Engineering Center (MRSEC), and in part by the Center for Materials Research and Analysis (CMRA).

The authors are with the Department of Physics and Astronomy and Center for Materials Research and Analysis, University of Nebraska, Lincoln, NE 68588 USA (e-mail: rskomski@unlserve.unl.edu).

Digital Object Identifier 10.1109/TMAG.2003.815746

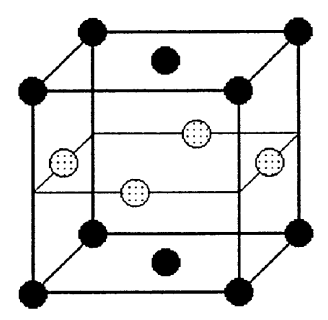

Fig. 1. L1 $1_{0}$ structure of PtCo magnets. The Pt (gray) and Co (black) atoms form alternating layers, which leads to considerable magnetocrystalline anisotropy, even if the c/a ratio is close to one.

law, where $\mathrm{n}=2$ for $\mathrm{K}_{1}$, and the corresponding finite-temperature expression $\hat{I}_{5 / 2}$ [9] yield a very similar dependence [4], [9].

However, one-sublattice model approaches are not able to describe complicated intermetallic alloys. For example, the transition-metal sublattice contributes comparatively little to the total anisotropy of rare-earth transition-metal intermetallics but is important to provide the interatomic exchange field necessary to realize the leading rare-earth anisotropy [4], [10], [11]. Here, we will show that a different two-sublattice mechanism is responsible for the finite-temperature magnetism of $3 d-4 d$ and $3 d-5 d$ $\mathrm{L} 1_{0}$ magnets.

\section{Atomic-SCALE CONSIDERATIONS}

Fig. 1 shows the tetragonal $\mathrm{L}_{0}$ structure. The Co atoms (black) ensure that the magnetization and the Curie temperature are reasonably high, whereas the Pt atoms (gray) provide the spin-orbit coupling from which the large anisotropy of the magnets results [4], [7]. However, to realize the Pt anisotropy it is necessary to have 1) a magnetic moment on the Pt atoms, so that the net spin-orbit interaction is nonzero, and 2) a sufficiently strong intersublattice exchange to stabilize the directions of the Pt moments at finite temperatures.

Elemental platinum is an exchanged-enhanced Pauli paramagnet and does not have a magnetic moment, but Pt atoms are easily spin-polarized by a suitable crystalline environment. Physically, $\uparrow$ and $\downarrow$ electrons hop from the Co atoms onto Pt sites and create a small Pt moment. A qualitative way of determining the Pt moment and the sign of the Pt-Co moment is to exploit the tight-binding moments theorem, which relates the bandstructure to the local atomic environment [12]-[14]. The analysis reveals that the magnetic Co atoms spin-polarize the Pt $5 \mathrm{~d}$ atoms, with ferromagnetic (FM) Co-Pt coupling $\mathrm{J}^{*}>0$. The Pt moment scales as $\mathrm{t} /(\mathrm{W}-\mathrm{I})$, where $\mathrm{t}$ is the Co-Pt nearest-neighbor hopping integral, $\mathrm{W}=1 / \mathrm{D}\left(\mathrm{E}_{\mathrm{F}}\right)$ is a Pt bandwidth parameter, and $\mathrm{I}$ is the intra-atomic Stoner exchange of the Pt atoms. 


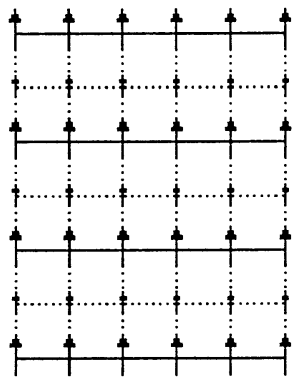

(a)

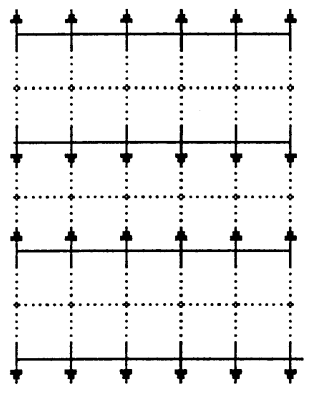

(b)
Fig. 2. Schematic spin structure: (a) FM and (b) AFM. Only in a FM environment, the Pt atoms exhibit a spin moment, but this moment is sufficient to realize magnetocrystalline anisotropy. The finite-temperature spin structure is random and, in a sense, intermediate between (a) and (b).

To investigate intra- and inter-atomic exchange effects on a first-principle level, we use the approach developed by Liechtenstein et al. [15]. A self-consistent spin-polarized linear muffin-tin orbital method [16] is used to calculate the magnetic moments and charge densities. To calculate the exchange interaction $\mathrm{J}_{\mathrm{ij}}$ between two different sites $\mathrm{i}$ and $\mathrm{j}$ we assume spherical charge and spin densities and employ the local force theorem, so that

$$
J_{i j}=\frac{1}{4 \pi} \sum_{L L^{\prime}} \operatorname{Im} \int_{-\infty}^{\varepsilon_{F}} d \varepsilon \Delta_{l}^{i}(\varepsilon) T_{L L^{\prime}}^{i j \uparrow}(\varepsilon) \Delta_{l^{\prime}}^{j}(\varepsilon) T_{L L^{\prime}}^{i j \downarrow}(\varepsilon) .
$$

Here, $T_{L L^{\prime}}^{i j \sigma}(\varepsilon)$ is the scattering path operator in the site $(\mathrm{i}, \mathrm{j})$ representation for different spin projections $(\sigma=\uparrow, \downarrow)$, and $\Delta_{l^{\prime}}^{j}(\varepsilon)=t_{i \uparrow}^{-1}-t_{i \downarrow}^{-1}$ is the difference of the inverse single-site scattering matrices.

The focus of the first-principle calculation is on the two spin configurations shown in Fig. 2, for which moments and exchange energies are calculated. Table I shows the obtained spin moments per atom. In the FM case, the Pt moment contains a d-electron contribution of $0.446 \mu_{B}$ and an sp-electron contribution of $-0.052 \mu_{B}$. The Co moment is very stable, changing by only $5.6 \%$ when going from the FM to the antiferromagnetic (AFM) configuration. By contrast, the FM Pt moment of nearly $0.4 \mu_{B}$ collapses in the an AFM configuration. The reason is that Co neighbors above and below the $\mathrm{Pt}$ atoms have opposite moments, so that the net exchange field acting the Pt 5d electrons and the $\mathrm{Pt}$ moment are zero.

In terms of the energy difference between the FM and AFM configurations, the exchange is $0.0031 \mathrm{Ry}$ per Co atom, corresponding to a temperature of $490 \mathrm{~K}$. This value and the moments presented in Table I serve as a first-principle input for the calculations in the following section.

Note that the values listed in Table I are spin moments. Except for Pt in the AFM configuration, both Pt and Co exhibit some orbital moment not specified by the present first-principle calculations. As the magnetocrystalline anisotropy, the orbital moment is a relativistic effect, but in the present context it is of little consequence, because the intersublattice exchange is a spin-only effect.
TABLE I

SPin Moments Per Atom For the FM And AFM Configurations of Fig. 2

\begin{tabular}{lll} 
Configuration & $\begin{array}{l}\text { Co moment } \\
\left(\mu_{\mathrm{B}}\right)\end{array}$ & $\begin{array}{l}\text { Pt moment } \\
\left(\mu_{\mathrm{B}}\right)\end{array}$ \\
\hline FM & 1.786 & 0.394 \\
$\mathrm{AFM}$ & 1.688 & 0 \\
\hline
\end{tabular}

\section{Finite-TeMPERAURE BeHAVIOR}

The finite-temperature anisotropy is obtained as a thermal average over all spin configurations. The starting point is the quasiclassical two-sublattice mean-field Hamiltonian

$$
H=H_{\mathrm{Co}}+H_{\mathrm{Pt}}+H^{*}
$$

where $H_{\mathrm{Co}}$ is the $3 \mathrm{~d}$ sublattice exchange, $H_{\mathrm{Pt}}$ describes the $\mathrm{Pt}$ atoms, and $H^{*}$ is the intersublattice interaction energy. In the present approximation, $H_{\mathrm{Co}}=-\mathrm{J}_{\mathrm{Co}-\mathrm{Co}} \mathbf{S} \cdot\langle\mathbf{S}\rangle$, where $\mathrm{S}$ is the cobalt spin moment and $\mathrm{J}_{\mathrm{Co}-\mathrm{Co}} \sim \mathrm{T}_{\mathrm{c}}$ is the cobalt intrasublattice exchange. The platinum sublattice Hamiltonian is

$$
\begin{aligned}
H_{\mathrm{Pt}}= & 1 / 2(\mathrm{~W}-\mathrm{I})\left(\mathrm{m}_{\mathrm{z}}^{2}+\mathrm{m}_{\mathrm{x}}^{2}+\mathrm{m}_{\mathrm{y}}^{2}\right) \\
& -\mathrm{K}_{\mathrm{o}}\left(\mathrm{m}_{\mathrm{z}}^{2}-\mathrm{m}_{\mathrm{x}}^{2} / 2-\mathrm{m}_{\mathrm{y}}^{2} / 2\right) .
\end{aligned}
$$

Here, $\mathrm{K}_{\mathrm{O}}$ is an anisotropy parameter which depends on the spinorbit coupling and on the crystal field. The interaction term is

$$
H^{*}=-\mathrm{J}^{*}\left(\mathrm{~m}_{\mathrm{z}} \cos \theta+\mathrm{m}_{\mathrm{x}} \sin \theta\right)\langle\mathrm{S}(\mathrm{T})\rangle
$$

where $\theta$ is the angle between the cobalt magnetization and the crystallographic $\mathrm{c}$ axis.

In the following, we ignore the weak dependence of the Co moment on the spin structure. The calculation of the free energy

$$
\mathrm{F}(\theta)=-\mathrm{k}_{\mathrm{B}} \mathrm{T} \ln \Sigma_{\mathrm{i}} \int \exp \left(-\mathrm{H}\left(\mathrm{S}_{\mathrm{i}}, \mathbf{m}, \theta\right) / \mathrm{k}_{\mathrm{B}} \mathrm{T}\right) \mathrm{d} \mathbf{m}
$$

is straightforward and yields the anisotropy

$$
\mathrm{K}_{1}(\mathrm{~T})=\mathrm{K}_{1}(0) \mathrm{M}_{\mathrm{Co}}^{2}(\mathrm{~T}) / \mathrm{M}_{\mathrm{Co}}^{2}(0) .
$$

In this equation, $\mathrm{J}^{*}$ enters the zero-temperature anisotropy $\mathrm{K}_{1}(0)=3 \mathrm{~K}_{\mathrm{o}} \mathrm{m}^{2} / 2$, where $\mathrm{m}=\mathrm{J}^{*} \mathrm{~S} /(\mathrm{W}-\mathrm{I})$, but not the temperature-dependent term. The temperature dependence of $\mathrm{K}_{1}$ originates from $\langle\mathrm{S}(\mathrm{T})\rangle$ in (4). Taking $\mathrm{K}_{1}(0)=5 \mathrm{MJ} / \mathrm{m}^{3}$ yields $\mathrm{K}_{\mathrm{o}}=21.5 \mathrm{MJ} / \mathrm{m}^{3}$. This large value is a result of the strong spin-orbit coupling of the Pr $5 \mathrm{~d}$ electrons.

Fig. 3 shows the temperature dependence of the leading $4 d / 5 d$ anisotropy contribution for $3 \mathrm{~d}$ spins of lengths $\mathrm{S}=1$ (solid line) and $\mathrm{S}=1 / 2$ (dashed line). The curves exhibit a pronounced negative curvature, which is favorable from the point of view of applications above room temperature, in contrast to the positive curvature of typical rare-earth transition-metal ferromagnets [4]. 


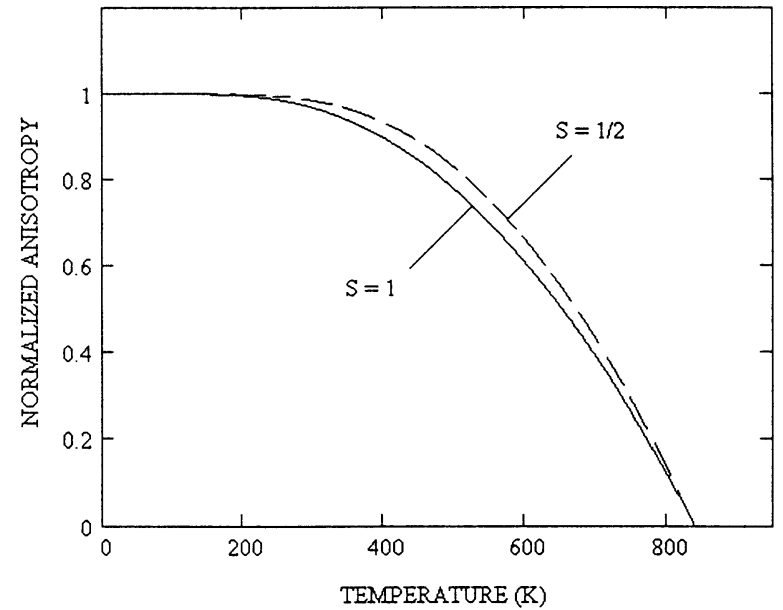

Fig. 3. Temperature dependence of the anisotropy of the $4 d / 5 d$ sublattice. $S=$ 1 is more appropriate for Co-containing $\mathrm{L} 1_{0}$ magnets.

Only very recently it has become possible to measure $\mathrm{K}_{1}(\mathrm{~T})$ of $\mathrm{L} 1_{0}$ magnets $(\mathrm{PtFe})$ over the whole range of temperature [17]. However, in [17] as well as in [18], ${ }^{1}$ the anisotropy is discussed in terms of a one-sublattice theory (Section I), which is a very crude approach.

\section{DISCUSSION AND CONCLUSION}

In spite of similarities with rare-earth transition-metal intermetallics, the temperature dependence of the anisotropy of PtCo exhibits some unique features. In rare-earth magnets, intersublattice interactions are important too, but rare-earth atoms obey Hund's rules and do not need an exchange field to develop a magnetic moment [11], [20]. The situation encountered in $\mathrm{L} 1_{0}$ magnets is, in a sense, comparable to that encountered in $3 d-5 f$ compounds such as uranium sulphide, where interatomic exchange is necessary to stabilize the uranium moment [21]. It is important to note that the present mechanism applies not only to PtCo but also to other magnetic $3 \mathrm{~d}-4 \mathrm{~d}$ and $3 \mathrm{~d}-5 \mathrm{~d}$ compounds crystallizing in the $\mathrm{L} 1_{0}$ structure, such as PdFe and PtFe.

In conclusion, the anisotropy of PtCo and its temperature dependence are due to Co $3 \mathrm{~d}$ electrons hopping onto Pt sites and spin-polarizing the Pt $5 \mathrm{~d}$ electrons. With increasing temperature, thermal disorder leads to a randomization of the direction of the Co spins and to a reduction of the exchange field

\footnotetext{
${ }^{1}$ A recent paper [19] uses a two-sublattice approach very similar to that of Section II but does not consider the temperature dependence of the anisotropy.
}

acting on the Pt atoms. As a consequence, the Pt moment, the net spin-orbit coupling, and the leading Pt anisotropy contribution collapse.

\section{REFERENCES}

[1] T. Klemmer, D. Hoydick, H. Okumura, B. Zhang, and W. A. Soffa, "Magnetic hardening and coercivity in $\mathrm{L} 1_{0}$ ordered FePd ferromagnets," Scripta Met. Mater, vol. 33, pp. 1793-1805, 1995.

[2] R. A. McCurrie, Ferromagnetic Materials-Structure and Properties. London, U.K.: Academic, 1994.

[3] D. J. Sellmyer, M. Yu, R. A. Thomas, Y. Liu, and R. D. Kirby, "Nanoscale design of films for extremely high density magnetic recording," Phys. Low-Dim. Struct., vol. 1-2, pp. 155-165, 1998.

[4] R. Skomski and J. M. D. Coey, Permanent Magnetism. Bristol, U.K.: Institute of Physics, 1999.

[5] S. Jeong, Y.-N. Hsu, M. E. McHenry, and D. E. Laughlin, "Structure and magnetic properties of $\mathrm{L}_{0} \mathrm{CoPt}(\mathrm{Ag} / \mathrm{MgO}, \mathrm{MgO})$ thin films," J. Appl. Phys., vol. 87, pp. 6950-6952, 2000.

[6] K. D. Belashchenko and V. P. Antropov, "Structure of macrodomain walls in polytwinned magnets," J. Appl. Phys., vol. 91, pp. 8474-8476, 2002 .

[7] G. H. O. Daalderop, P. J. Kelly, and M. F. H. Schuurmans, Ultrathin Magnetic Structures I, J. A. C. Bland and B. Heinrich, Eds. Berlin, Germany: Springer-Verlag, 1994, pp. 40-65.

[8] R. F. Sabiryanov and S. S. Jaswal, "Electronic structure and magnetic properties of hard/soft multilayers," J. Magn. Magn. Mater., vol. 177-181, pp. 989-990, 1998.

[9] E. R. Callen and H. B. Callen, "Static magnetoelastic coupling in cubic crystals," Phys. Rev., vol. 129, pp. 578-593, 1963.

[10] J. M. D. Coey, Ed., Rare-Earth Iron Permanent Magnets. Oxford, U.K.: Oxford Univ. Press, 1996.

[11] R. Skomski, "Finite-temperature behavior of anisotropic two-sublattice magnets," J. Appl. Phys., vol. 83, pp. 6724-6726, 1998.

[12] F. Cyrot-Lackmann, "Sur le calcul de la cohésion et de la tension superficielle des métaux de transition par une méthode de liaisons fortes," $J$. Phys. Chem. Solids, vol. 29, pp. 1235-1243, 1968.

[13] A. P. Sutton, Electronic Structure of Materials. Oxford, U.K.: Oxford Univ. Press, 1993.

[14] R. Skomski, "Magnetoelectric Néel anisotropies," IEEE Trans. Magn., vol. 34, pp. 1207-1209, July 1998.

[15] A. I. Liechtenstein, M. I. Katsnelson, V. P. Antropov, and V. A. Gubanov, "Local spin density functional approach to the theory of exchange interactions in ferromagnetic metals and alloys," J. Magn. Magn. Mater., vol. 67, pp. 65-74, 1987.

[16] O. K. Andersen, "Linear methods in band theory," Phys. Rev. B, vol. 12, p. 3060, 1975.

[17] N. H. Hai, N. M. Dempsey, and D. Givord, "Magnetic properties of hard FePt prepared by cold deformation," IEEE Trans. Magn., vol. 39, pp. 2914-2916, 2003.

[18] O. Mraysov, private communication, 2003.

[19] H. Zeng, R. Sabirianov, O. Mryasov, M. L. Yan, K. Cho, and D. J. Sellmyer, "Curie temperature of FePt: $\mathrm{B}_{2} \mathrm{O}_{3}$ nanocomposite films," Phys. Rev. B, vol. 66, pp. 184425-1-184425-6, 2002.

[20] N. H. Duc, T. D. Hien, D. Givord, J. J. M. Franse, and F. R. de Boer, "Exchange interactions in rare-earth transition-metal compounds," J. Magn. Magn. Mater, vol. 124, pp. 305-311, 1993.

[21] R. Skomski, "Exchange-controlled magnetic anisotropy," J. Appl. Phys., vol. 91, pp. 8489-8491, 2002. 